\title{
ON AMBIGUITY DETECTION AND POSTPROCESSING SCHEMES USING CLUSTER ENSEMBLES
}

\author{
Amparo Albalate, Aparna Suchindranath, Mehmet Muti Soenmez \\ Institute of Information Technology, University of Ulm \\ amparo.albalate@uni-ulm.de,aparna.suchindranath@uni-ulm.de,mehmez.soenmez@uni-ulm.de \\ David Suendermann \\ SpeechCycle Labs, NY, USA \\ david@speechcycle.com
}

Keywords: Cluster Ensembles, Uncertainty, Ambiguity.

\begin{abstract}
In this paper, we explore the cluster ensemble problem and propose a novel scheme to identify uncertain/ambiguous regions in the data based on the different clusterings in the ensemble. In addition, we analyse two approaches to deal with the detected uncertainty. The first, simplest method, is to ignore ambiguous patterns prior to the ensemble consensus function, thus preserving the non-ambiguous data as good "prototypes" for any further modelling. The second alternative is to use the ensemble solution obtained by the first method to train a supervised model (support vector machines), which is later applied to reallocate, or "recluster" the ambiguous patterns. A comparative analysis of the different ensemble solutions and the base weak clusterings has been conducted on five data sets: two artificial mixtures of five and seven Gaussian, and three real data sets from the UCI machine learning repository. Experimental results have shown in general a better performance of our proposed schemes compared to the standard ensembles.
\end{abstract}

\section{INTRODUCTION}

In supervised learning, an ensemble is a combination of classifiers with the goal to improve the robustness and accuracy of the constituents classifiers. To date, a large body of research on classifier ensembles has been conducted, showing important improvements in comparison to single classifiers (Schapire, 2002, Strehl et al., 2002, Kuncheva, 2004).

In recent years, the achievements attained in the field of supervised learning have increasingly attracted the attention of the unsupervised community, and subsequently the ensemble framework has also been investigated for clustering tasks. A comprehensive overview has been provided in (Strehl et al., 2002).

As in supervised learning, different scenarios to achieve the required diversity of components in a cluster ensemble have been proposed in the literature. According to (Strehl et al., 2002) three main approaches can be distinguished:

The Feature Distributed Clustering (FDC) approach is to run a clustering algorithm on a common set of objects but different partial views of the feature space.

The Object distributed clustering (ODC), or resampling, is a second diversifying approach, where the clusterers are fed with a common set of features but different subsets of the data objects.

Finally, in the Robust Centralised clustering $(R C C)$ scenario, the ensemble components are achieved by applying a common set of objects and features to different clustering algorithms, using either a unique or different distance functions.

The problem of combining the component partitions in the ensemble to obtain an appropriate aggregate solution is formulated as to optimise a given consensus function. Different heuristics have been proposed in (Strehl et al., 2002) for achieving the mentioned consensus function: the cluster similarity partitioning algorithm (CPSA), the hypergraph partitioning algorithm (HGPA) and the metaclustering algorithm (MCLA). In this paper, we focus on the CPSA heuristic, in which the different clusterings are merged in a so-called co-association matrix, whose $(i, j)$ entries encode the agreement of the different partitions on clustering together the input objects $i$ 
and $j$. Hence, the co-association matrix is a similarity matrix which can be again applied to a clustering algorithm to recluster the dataset.

The main objective of this paper is to exploit the redundancy of clusterings in the ensemble for detecting ambiguous regions in the data with a high degree of uncertainty. Ambiguities can be associated to different factors. For example, a high proximity of two or more underlying classes may produce a certain overlap between clusters, specially at patterns close to the class' boundaries. Note that this kind of ambiguities, in contrast to outliers, is not due to abnormal deviations with respect to the rest of the patterns in the dataset, but inherently caused by the underlying class structure. The detection and postprocessing of outlier patterns is an important area in artificial intelligence, with numerous research contributions. However, previous work to detect ambiguities has a more limited coverage in comparison to the outliers literature. For example, in (Lin et al., 2006), an extension of binary support vector machines was proposed to identify new classes corresponding to uncertain regions. In this work, we used the terms ambiguity or uncertainty to indicate such patterns with a high probability of belonging to a different cluster than the one they are assigned to. Our assumption is that the ambiguous regions should reflect a low agreement between the clusterers in the ensemble. In the following sections we explain how to detect ambiguities based on this idea. Following the detection of ambiguities, we propose a strategy to analyse ambiguous data. The simplest approach is to ignore these regions, focusing on the rest of patterns as good prototypes. The second approach is to assist the cluster ensemble with the help of a robust "supervised" classification method: Support Vector Machines (SVMs). We explain how SVMs are used in an unsupervised manner to solve the ambiguity problem. Finally, we show the improvements in comparsion to the basic ensemble by discarding ambiguous patterns, and even after "reclustering" these patterns with the help of SVMs.

The structure of this paper is as follows: In Section 2 the analysed data sets are presented, in Section 3. we describe the cluster ensemble approach used in this work. The detection and post-processing of ambiguities are explained in Sections 4 and 5 respectively. Finally, we show evaluation results in Section 6 and draw conclusions in Section 7

\section{DATA SETS}

In this work, we used five different datasets: two mixtures of Gaussians, and three real data sets from



(a)



(b)
Figure 1: Mixture of Gaussians data-sets. 1(a) Five Gaussians, 1(b) seven Gaussians

the UCI machine learning repository.

Mixtures of Gaussians These data sets comprise two mixtures of five and seven Gaussians with 1250 and 1750 points in two dimensions (Figure 1), where a certain number of overlapping patterns (potential ambiguities) can be observed. We used this data sets with the purpose to provide an example of the uncertainty problem in cluster ensembles.

Wine data set (WINE) The wine set is one of the popular data sets from the UCI databank. It consists of 178 instances with 13 attributes, representing three different types of wines.

Wisconsin breast cancer data set (BREAST) This data set constains 569 instances in 10 dimensions, with 10 different features extracted from digitised images of breast masses. The two existing classes are referred to the possible breast cancer diagnosis (malignant, benign).

Handwritten digits data set (PENDIG) The third real data set is for pen-based recognition of handwritten digits. In our experiments, we used the test partition, composed of 3498 samples with 16 attributes. Ten classes can be distinguished for the digits $0-9$.

\section{CLUSTER ENSEMBLES}

Diversifying scenario In order to achieve the required diversity of partitions in the ensemble, a robust centralised clustering scenario (RCC) has been selected, using four different clustering methods: the partition around medoids (pam), and the complete, average and centroid linkage algorithms. Each clustering method has been provided with the target number of clusters $k$, which is assumed to be known. Our library or pool of clusterings is thus composed of four 
component partitions, obtained by applying the four mentioned clustering algorithms to the matrix of Euclidean distances between the data objects. In the following, we refer to the clustering algorithms applied to the raw Euclidean dissimilarities as base clusterers.

Consensus function A CSPA consensus function has been applied to the ensemble partitions in order to compute an aggregate cluster solution. Fist, the CSPA algorithm derives the co-association matrix, $\mathcal{A}$, whose elements $A_{i j}$ denote the number of times that the objects $i$ and $j$ in the dataset have been assigned to the same cluster by any pair of base clusterers in the ensemble. As for the final consensus clustering, a comparative analysis has been performed by applying again any of the aforementioned clustering methods, initially used as based clusterers, to cluster the co-association matrix. At this stage, the clustering algorithms are refered to as consensus clusterers, in order to be distinguished from their respective previous roles as base clusterers. The decision for a unique consensus clustering has not been addressed in this paper. However, one option would be to use a supra consensus by selecting the clustering with highest average normalised mutual information (ANMI), in a similar way as suggested in (Strehl et al., 2002) for choosing between different consensus heuristics.

Because the agglomerative and pam clustering algorithms used in this work are based on dissimilarity functions and the co-association matrix naturally represents similarities between objects, a conversion of the co-association values has been performed as follows:

$$
A_{i j}^{\prime}=1-\frac{A_{i j}}{\max (\mathcal{A})}
$$

so that the new co-association values $\mathcal{A}^{\prime}$ denote distances between the objects.

\section{DETECTION OF AMBIGUOUS REGIONS}

In this section, we describe the approach used to detect uncertain regions in a data set given the component partitions in the ensemble. First, we need to quantify the "uncertainness" of each data point. As explained in Section 1, we assume ambiguous patterns should lead to a lower consensus between the ensemble partitions. Thus, the first goal is to measure the agreement on which a given pattern is consistently placed into the same cluster by the different base clusterers in the ensemble. The solution to this problem is not straightforward, given that the labels rendered by a clustering algorithm are virtual labels and cannot be directly compared. We propose a solution based on the concept of mutual information (Cover and Thomas, 1991) between different partitions. The normalised mutual information (NMI) was proposed in (Strehl et al., 2002) as a measure of the consensus between two cluster solutions, $\lambda^{(a)}$ and $\lambda^{(n)}$, (Equation 2).

$$
\begin{aligned}
& \operatorname{NMI}\left(\lambda^{(a)}, \lambda^{(b)}\right)= \\
& =\frac{\sum_{h=1}^{k(a)} \sum_{l=1}^{k(b)} n_{h, l} \log \left(\frac{n \cdot n_{h, l}}{n_{h}^{(a)} n_{l}^{(b)}}\right)}{\sqrt{\left(\sum_{h=1}^{k(a)} n_{h}^{(a)} \log \left(\frac{n_{h}^{(a)}}{n}\right)\right)\left(\sum_{l=1}^{k(b)} n_{l}^{(b)} \log \left(\frac{n_{l}^{(b)}}{n}\right)\right)}}
\end{aligned}
$$

Denoting $n$, the number of observations in the dataset, $k(a)$ and $k(b)$, the number of clusters in the partitions $\lambda^{(a)}$ and $\lambda^{(b)} ; n_{h}^{(a)}$ and $n_{l}^{(b)}$, the number of elements in the clusters $C_{h}$ and $C_{l}$ of the partitions $\lambda^{(a)}$ and $\lambda^{(b)}$ respectively, and $n_{h, l}$, the number of overlapping elements between the clusters $C_{h}$ and $C_{l}$.

For the present task, we measure the degree of overlap between the clusters $C_{h}^{(a)}$ and $C_{l}^{(b)}$ containing a pattern $p$ under evaluation in the partitions $\lambda^{(a)}$ and $\lambda^{(b)}$, respectively. We call this metric the Normalised Cluster Overlap (NCO):

$$
\operatorname{NCO}\left(\lambda^{(a)}, \lambda^{(b)}, p\right)=\frac{n_{h, l}}{\sqrt{\left(n_{h}^{(a)} \log \frac{n_{h}^{(a)}}{n}\right)\left(n_{l}^{(b)} \log \frac{n_{l}^{(b)}}{n}\right)}}
$$

The overall agreement in clustering the pattern is then defined as the accumulated sum of $N C O$ s considering all possible pairs of cluster partitions in the ensemble:

$$
A N C O(\lambda, p)=\sum_{r=1}^{L-1} \sum_{r^{\prime}=r+1}^{L} \operatorname{NCO}\left(\lambda^{(r)}, \lambda^{\left(r^{\prime}\right)}, p\right)
$$

Because $A N C O$ is a measure of consensus, low values correspond to patterns with higher uncertainty and vice-versa.

Figure 3 shows an example of the patterns identified as ambiguities (in red colour) using the above described approach on the mixtures of Gaussians.

Finally, an ambiguous pattern is detected if its $A N C O$ value is found below a given threshold $A N C O_{t h}$. In this work, the threshold value has been determined by visualising the histogram plot of the $A N C O$ values. Figure 2 shows the histograms of $A N C O$ values in the analysed data sets and illustrates 


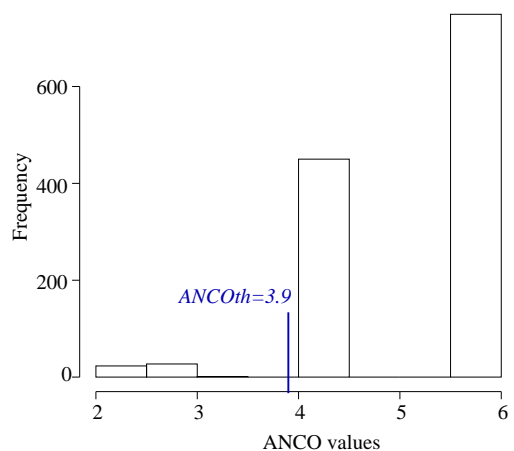

(a)



(b)

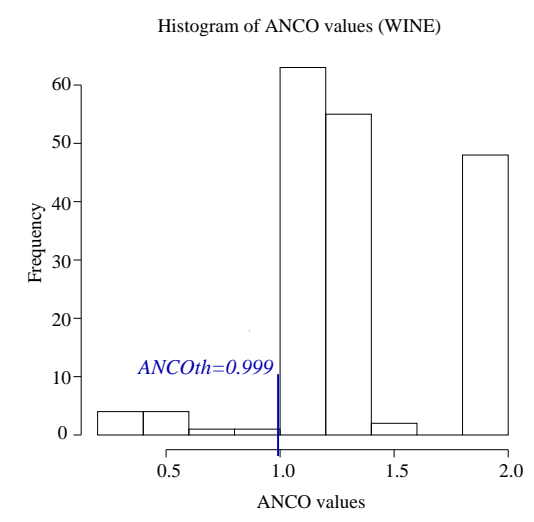

(c)

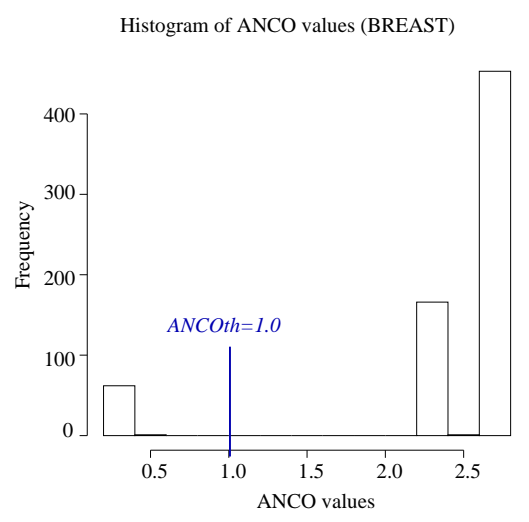

(d)

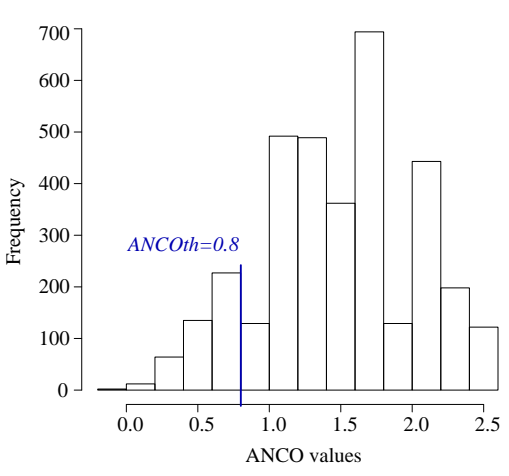

(e)

Figure 2: Histogram plots used in the determination of the ANCO threshold in the evaluated data sets.

the criterion for selecting the $A N C O$ thresholds corresponding to ambiguities. (Future work is to automate this step).

\section{PROCESSING AMBIGUOUS DATA}

\subsection{Support Vector Machines (SVM)}

Support Vector Machines are amongst the most popular classification and regression algorithms because of their robustness and good performance in comparison to other classifiers (Burges, 1998. Joachims, 1998. Lin et al., 2006). In its basic form, SVM were defined for binary classification of linearly separable data. Let us denote a set of $L$ training patterns $\mathcal{X}=$ $\left\{x_{1}, x_{2}, \cdots x_{L}\right\}$ in $\mathcal{R}^{D}$. For binary classes, we denote the set of labels corresponding to the training data: $\mathcal{Y}=\left\{y_{1}, \cdots, y_{L}\right\}$, with $y_{i} \in\{1,-1\}$ (Figure 4).

Assuming that the classes $(+1,-1)$ are linearly separable, the SVM goal is to orientate a hyperplane $H$ which maximises the margin between the closest members of the two classes (also called support vec- tors). The searched hyperplane is given by the equation:

$$
H=\left\{x \in \mathcal{R}^{D} \mid w x+b=0\right\}
$$

denoting $w$ the normal vector of the hyperplane. In addition, the parallel hyperplanes $H_{1}$ and $H_{2}$ at the support vectors of classes $y=1$ and $y=-1$ are defined as:

$$
\begin{aligned}
& H_{1}=\left\{x \in \mathcal{R}^{D} \mid w x+b=1\right\},(y=1) \\
& H_{2}=\left\{x \in \mathcal{R}^{D} \mid w x+b=-1\right\},(y=-1)(6)
\end{aligned}
$$

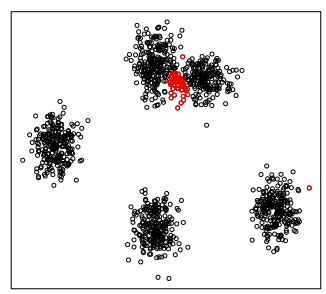

(a)



(b)
Figure 3: Example of detected ambiguities in the mixtures of Gaussians. Ambiguous patterns are depicted with red circles, in contrast to non-ambiguous patterns(black circles). 3(a) Five Gaussians, 3(b) seven Gaussians 


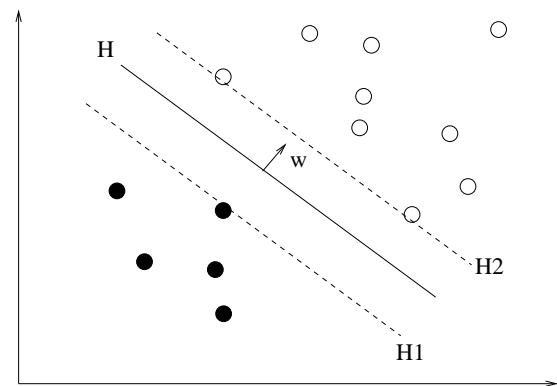

Figure 4: SVM example of binary classification through hyperplane separation (in this two-dimensional case the hyperplane becomes a line).

It can be demonstrated that the margin between the hyperplanes $H_{1}$ and $H_{2}$ is $\frac{1}{\|w\|}$. In addition, the training points at the left/right sides of the hyperplanes $H_{1}$ and $H_{2}$ need to satisfy:

$$
y_{i}\left(w x_{i}+b\right)-1 \geq 0 \quad \forall i
$$

Thus, the maximum margin hyperplane is obtained by solving the following objective:

$$
\text { minimise }\|w\| \text { such that } y_{i}(w x+b)-1 \geq 0
$$

By applying Lagrange multipliers, the objective in Equation 9 can be solved using constrained quadratic optimisation. After some manipulations, it can be shown that the initial objective is equivalent to:

$$
\begin{array}{ll}
\text { maximise } & \sum_{i=1}^{L}\left(\alpha_{i}-\frac{1}{2} \sum_{j} \alpha_{i} \alpha_{j} y_{i} y_{j} x_{i} x_{j}\right) \\
\text { subject to } & \alpha_{i} \geq 0 \forall i, \text { and } \sum_{i=1}^{L} \alpha_{i} y_{i}=0
\end{array}
$$

The solution of this quadratic optimisation problem is a set of coefficients $\alpha=\left\{\alpha_{1}, \cdots, \alpha_{L}\right\}$ which are finally applied to calculate the hyperplane variables $w$ and $b$ :

$$
\begin{aligned}
w & =\sum \alpha_{i} y_{i} x_{i} \\
b & =\frac{1}{N_{s}} \sum_{s \in \mathcal{S}} y_{s}-\sum_{m \in \mathcal{S}} \alpha_{m} y_{m} x_{m} x_{s}
\end{aligned}
$$

where $\mathcal{S}$ denotes the set of support vectors, of size $N_{S}$

Although the solution in Equation [10 is found for the basic problem of binary, linearly separable classes, SVMs have been extended for both multiclass and non-linear problems.
The kernel trick for non-linearly separable classes The application of SVMs to non-linearly separable classes is achieved by substituting the dot product $x_{i} x_{j}$ in Equation 9 by an appropriate function, the so called kernel $k\left(x_{i}, x_{j}\right)$. The purpose of this "kernel trick" is that a non-linear kernel can be used to transform the feature space into a new space of higher dimension. In this high dimensional space it is possible to find a hyperplane to separate classes which may not be originally separable in the initial space. In other words, the kernel function is equivalent to the dot product:

$$
k\left(x_{i}, x_{j}\right)=<\phi\left(x_{i}\right) \phi\left(x_{j}\right)>
$$

where $\phi$ denotes a mapping of a pattern into the higher dimensional space. The main advantage is that the kernel computes these dot products without the need to specify the mapping function $\phi$.

Multi-class classification The extension for the multi-class problem is achieved through a combination of multiple SVM classifiers. Two different schemes have been proposed to solve this problem: in a one-against-all approach, $k$ hyperplanes are obtained to separate each class from the rest of classes. In a one-against-one approach, $\left(\begin{array}{l}k \\ 2\end{array}\right)$ binary classifiers are trained to find all possible hyperplanes to separate each pair of classes.

\subsection{SVMs to recluster ambiguous data}

The simplest approach to deal with uncertainty is to treat the ambiguous patterns as noise patterns and ignore them, retaining the rest of data as good prototypes for any further processing. However, rejecting uncertain data can result in a considerable loss of relevant information, specially when these observations can help reveal the underlying data distribution. Therefore, in this section we propose an alternative to the simple rejection of the detected ambiguities based on support vector machines.

First, patterns detected as ambiguous (Section 4) are removed from the ensemble partitions. The coassociation matrix is recalculated considering only the unambiguous patterns. Intuitively, the recalculated co-association matrix should reflect higher consensus among the combined partitions, as the removed ambiguous patterns introduced a certain "noise" in this matrix. The consensus clustering is then applied to the new co-association matrix in order to achieve a more robust aggregate solution. If no further post-processing of the removed ambiguities is performed, the described procedure up to this stage corresponds to the simple removal of ambiguities. 
Processing of ambiguities Now, we consider the consensus solution obtained by using any of the aforementioned clustering algorithms to recluster the new co-association matrix after removal of ambiguities. Since this solution is achieved in absence of ambiguous patterns, we assume a more robust representation of the surrogate classes is attained in the output clusters. Of course, a certain error is made by the clustering process, which can be measured if reference class labels are available for a dataset, by calculating the normalised mutual information (NMI) between the cluster solution and the reference labels. However, we ignore this error, assuming that the class structure is adequately covered in the cluster solution. Next, we assign a different "virtual" label to each one of the obtained clusters - or classes. Thereby, a training set is implicitely generated in an unsupervised manner, using only the information in the ensemble - the only supervised action involved in the whole process is the selection of an $A N C O$ threshold for detecting ambiguous patterns, but we have shown (Section 4) how this threshold can be easily determined by using histograms. This automatically generated training set is then used to train a model based on Support Vector Machines to find the hyperplanes separating the (virtual) classes. Finally, the SVM model is applied to make predictions on the ambiguous patterns, previously removed from the ensemble. Hence, the SVM decides which cluster in the consensus solution an ambiguous pattern should be reallocated to.

\section{EVALUATION AND TESTS}

For evaluation purposes, we compared the clustering solutions with the reference category labels, which are available for all analysed data sets. There are different external validation metrics which can be used to measure the correspondence between a cluster partition and the reference labels, including entropy, purity (Boley et al., 1999), or the Normalised Mutual Information (NMI, Equation 2). In this paper we selected the latter one due to its property of impartiality versus the number of clusters, in contrast to entropy or purity, as suggested by Stern and Gosh (Strehl et al., 2002).

We thus compared the NMI-based quality of the ensemble consensus solutions (by using the agglomerative and pam algorithms as different consensus clusterers applied to the co-association matrix) with the values obtained by their respective base clusterers. In addition, as is the focus of this work, we also evaluated the final ensemble solution when our scheme to tackle ambiguities is introduced. In this respect, two situations have been considered: (a) simple removal of ambiguous patterns (in which case the category labels corresponding to ambiguities have been also removed from the reference label sets prior to test), and (b) post-processing ambiguities with the help of support vector machines.

Tables 1 to 5 show the results obtained with the evaluated approaches on the mixtures of Gaussians, PENDIG, BREAST and WINE data sets. The first rows show NMI values obtained by the base clusterers (the complete, average and centroid linkages and the partitioning around medoids applied on the original matrix of object distances (ensemble components). The second rows refer to the aggregate ensemble solutions obtained by applying again the initial clustering algorithms (complete, average, centroid linkage and pam) as different consensus clusterers used to recluster the co-association matrices. The third rows indicate the performance of the ensembles when the ambiguity detection (AD) schemes are applied and the ambiguous patterns are removed prior to consensus clustering. Finally, the fourth and last rows show the NMI values obtained by the final ensemble solutions when the AD is introduced to detect ambiguities and Support Vector Machines models are applied to postprocess and reallocate ambiguous data, using radial and linear kernel functions (referred to as svm R and $\mathrm{L}$, respectively). Note also that the last columns in each row refer to the average NMI scores of the four clustering algorithms in each case.

As it can be observed, the ensemble approach outperforms the corresponding base clusterers in all data sets except WINE. The poorer performance in this dataset can be associated to the inability of two of the base clusterers (50\% of the ensemble components) in recovering any class structure (NMI values lower than $0.40 \%)$. This considerable proportion $(50 \%$ of the ensemble components) of "bad" clusterings has an impact on the new co-association matrix, in such a way that a third agglomerative approach fails to achieve an adequate consensus, although the same algorithm was originally able to recover more than $50 \%$ of the class structure (NMI score) by using the object distance matrix. On the other hand, note that the consensus based on the partitioning around medoids algorithm (pam) outperforms the corresponding base clusterer in the ensemble (pam applied to original distances), which also shows the best performance among the base clusterers.

Nevertheless, the ensemble solutions outperform the base components in all other data sets, where at least $3 / 4$ of the ensemble components are able to detect some class structure (NMI values greater than $50 \%)$. The robustness of the ensemble solution is 
Table 1: Seven Gaussians data set.

\begin{tabular}{lccccc}
\hline & \multicolumn{4}{c}{ Clustering } & \multirow{2}{*}{ Avg. } \\
\cline { 2 - 5 } & com. & avg. & cen. & Pam & \\
\hline Base & 0.907 & 0.913 & 0.915 & 0.945 & 0.920 \\
Ensemble(E) & 0.924 & 0.921 & 0.924 & 0.921 & 0.922 \\
E + AD(ignore) & 0.956 & 0.956 & 0.956 & 0.956 & 0.956 \\
E + AD(svm L) & 0.939 & 0.939 & 0.939 & 0.939 & 0.939 \\
E + AD(svm R) & 0.938 & 0.938 & 0.938 & 0.938 & 0.938 \\
\hline
\end{tabular}

Table 2: Five Gaussians data set.

\begin{tabular}{lccccc}
\hline & \multicolumn{4}{c}{ Clustering } & \multirow{2}{*}{ Avg. } \\
\cline { 2 - 5 } & com. & avg. & cen. & Pam & \\
\hline Base & 0.955 & 0.932 & 0.908 & 0.943 & 0.935 \\
Ensemble & 0.943 & 0.945 & 0.945 & 0.945 & 0.944 \\
E + AD(ignore) & 0.974 & 0.974 & 0.974 & 0.974 & 0.974 \\
E. + AD(svm L) & 0.950 & 0.950 & 0.950 & 0.950 & 0.950 \\
E. + AD(svm R) & 0.949 & 0.949 & 0.949 & 0.949 & 0.949 \\
\hline
\end{tabular}

Table 3: PENDIG data set.

\begin{tabular}{lccccc}
\hline & \multicolumn{4}{c}{ Clustering } & \multirow{2}{*}{ Avg. } \\
\cline { 2 - 5 } & com. & avg. & cen. & Pam & \\
\hline Base & 0.537 & 0.652 & 0.030 & 0.658 & 0.469 \\
Ensemble (E) & 0.324 & 0.617 & 0.331 & 0.679 & 0.488 \\
E + AD(ignore) & 0.544 & 0.673 & 0.562 & 0.730 & 0.627 \\
E + AD(svm L) & 0.507 & 0.632 & 0.517 & 0.690 & 0.587 \\
E + AD(svm R) & 0.512 & 0.638 & 0.529 & 0.692 & 0.593 \\
\hline
\end{tabular}

also revealed by smaller standard deviations of NMI scores across different consensus clusterings, in comparison to the respective base clusterings.

The incorporation of our approach to deal with ambiguities shows important improvements with respect to the standard ensemble. The best performance is attained by detecting and rejecting ambiguous patterns. The average increment of NMI scores ranges from only one percentual point in the WINE dataset, to $8 \%$ or $14 \%$ in the BREAST and PENDIG data sets.

Also the reallocation of ambiguities using support vector machines results in higher NMI scores when compared to the initial ensemble. Different average improvements are observed, up to $4.3 \%$ and $9.9 \%$ in the BREAST and PENDIG data sets, respectively.

\section{CONCLUSIONS AND FUTURE DIRECTIONS}

In this paper we have explored the cluster ensemble problem, which aims at combining different cluster partitions in order to improve the performance
Table 4: BREAST data set

\begin{tabular}{lccccc}
\hline & \multicolumn{4}{c}{ Clustering } & \multirow{2}{*}{ Avg. } \\
\cline { 2 - 5 } & com. & avg. & cen. & Pam & \\
\hline Base & 0.520 & 0.677 & 0.018 & 0.741 & 0.489 \\
Ensemble & 0.685 & 0.685 & 0.685 & 0.685 & 0.685 \\
E+ AD(ignore) & 0.766 & 0.766 & 0.766 & 0.766 & 0.766 \\
E+ AD(svm L) & 0.726 & 0.726 & 0.726 & 0.726 & 0.726 \\
E+ AD(svm R) & 0.728 & 0.728 & 0.728 & 0.728 & 0.728 \\
\hline
\end{tabular}

Table 5: WINE data set.

\begin{tabular}{lccccc}
\hline & \multicolumn{4}{c}{ Clustering } & \multirow{2}{*}{ Avg. } \\
\cline { 2 - 5 } & com. & avg. & cen. & Pam & \\
\hline Base & 0.550 & 0.031 & 0.038 & 0.721 & 0.335 \\
Ensemble(E) & 0.031 & 0.031 & 0.031 & 0.729 & 0.205 \\
E + AD(ignore) & 0.039 & 0.039 & 0.039 & 0.745 & 0.215 \\
E + AD(svm L) & 0.038 & 0.038 & 0.038 & 0.737 & 0.213 \\
E + AD(svm R) & 0.038 & 0.038 & 0.038 & 0.737 & 0.213 \\
\hline
\end{tabular}

and robustness of the aggregate solutions in comparison to the ensemble components. In particular, we focused on the Cluster Similarity Partitioning scenario (CSPA). This approach to cluster ensembles is to combine the clusterings by calculating an intermediate co-association matrix, which encodes the consensus or agreement between the partitions in the ensemble. The co-association matrix is used by any clustering algorithm to provide a higher-level, consensus clustering of the input data.

We further incorporate a strategy that is able to detect ambiguous regions in the data by analysing the different partitions in the ensemble. Prior to the consensus clustering, the ambiguities detected are removed from the component partitions, resulting in notable improvements of the aggregate solutions in comparison to the standard ensemble. We also propose an approach to reallocate an ambiguous pattern into one of the output clusters by using support vector machines in an unsupervised manner. An improvement of the ensemble performance has also been observed in this case.

Future work is to increase the number of clusterers in the ensemble and investigate ensemble selection approaches (Fern and Lin, 2008) in order to avoid the potential degradation of the ensemble performance if a significant number of "bad" clusterers inappropriate for a dataset are present among the ensemble components.

\section{REFERENCES}

Boley, D., Gini, M., Gross, R., Han, E.-H., Karypis, G., Kumar, V., Mobasher, B., Moore, J., and Hastings, K. (1999). Partitioning-based clustering for web docu- 
ment categorization. Decis. Support Syst., 27(3):329341.

Burges, C. J. C. (1998). A tutorial on support vector machines for pattern recognition. Data Mining and Knowledge Discovery, 2:121-167.

Cover, T. M. and Thomas, J. A. (1991). Elements of Information Theory. John Wiley \& sons.

Fern, X. Z. and Lin, W. (2008). Cluster ensemble selection. In Proceedings of the SIAM International Conference on Data Mining, pages 787-797.

Joachims, T. (1998). Text categorization with support vector machines: learning with many relevant features. In Proceedings of ECML-98, 10th European Conference on Machine Learning, number 1398, pages 137-142.

Kuncheva, L. I. (2004). Classifier ensembles for changing environments. In Multiple Classifier Systems, pages 1-15. Springer.

Lin, Y.-M., Wang, X., Ng, W., Chang, Q., Yeung, D., and Wang, X.-L. (2006). Sphere classification for ambiguous data. In Proceedings of International Conference on Machine Learning and Cybernetics, pages 25712574.

Schapire, R. E. (2002). The boosting approach to machine learning: An overview. In Proceedings of the 2002 MSRI Workshop on Nonlinear Estimation and Classification, pages 149-173. Springer.

Strehl, A., Ghosh, J., and Cardie, C. (2002). Cluster ensembles - a knowledge reuse framework for combining multiple partitions. Journal of Machine Learning Research, 3:583-617. 